\title{
The Dangers of Distal Airway Endotracheal Suction Trauma
}

\section{Mr W.O. Bennett, Mr J.A. Gaskin, Dr P.A. Stoddart and Mr M.W. Saunders \\ Bristol Royal Hospital For Children, Bristol, U.K. \\ Email: warben@gmail.com}

\begin{abstract}
A 28 week old premature male twin was referred to the regional neonatal intensive care unit with lobar collapse presumed to be caused by mucus plugging. We describe the management and finding of a bronchial granuloma likely secondary to distal airway suction trauma.
\end{abstract}

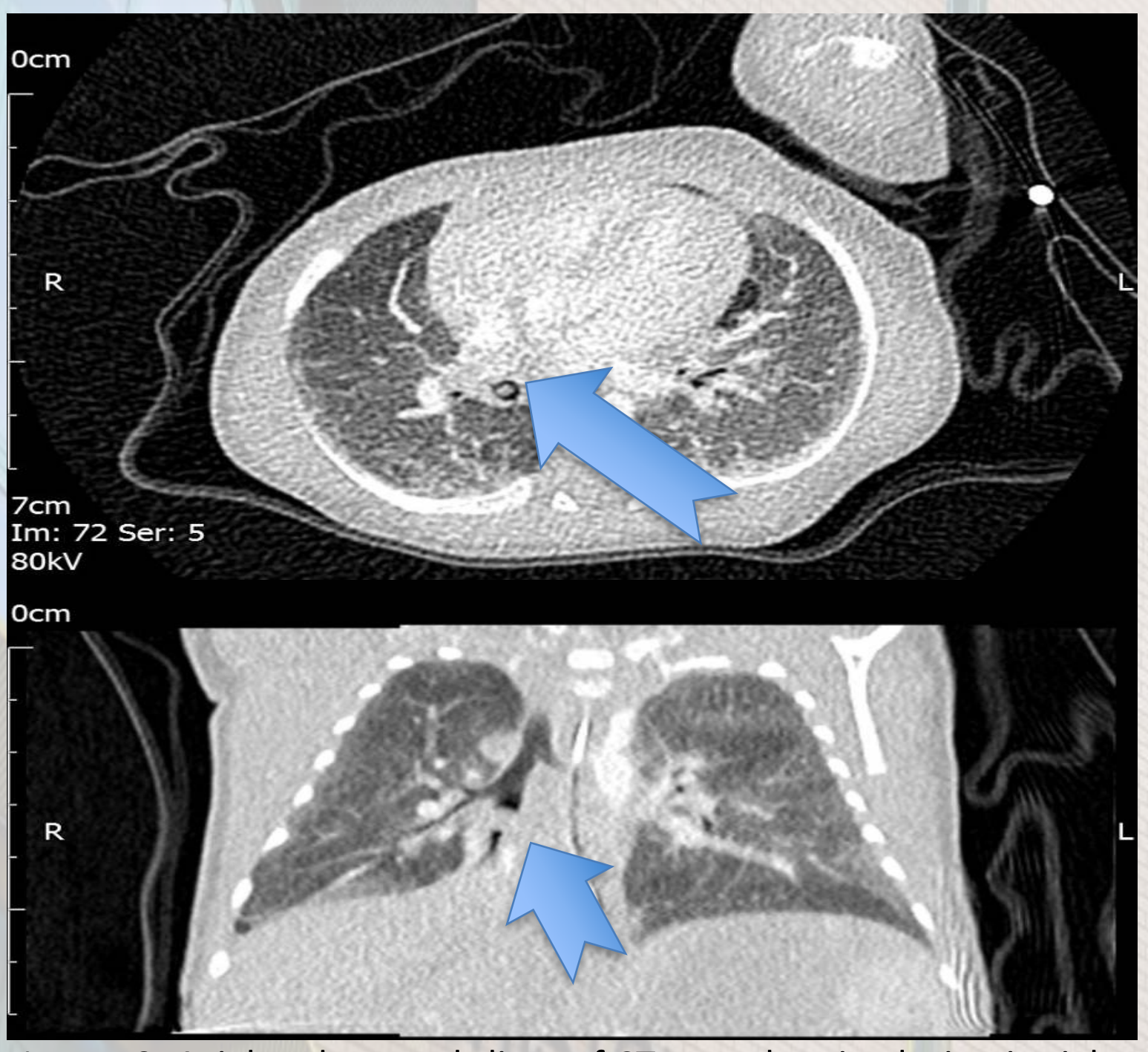

Image 2: Axial and coronal slices of CT scan showing lesion in right lower main bronchus. Originally thought to be a mucus plug.

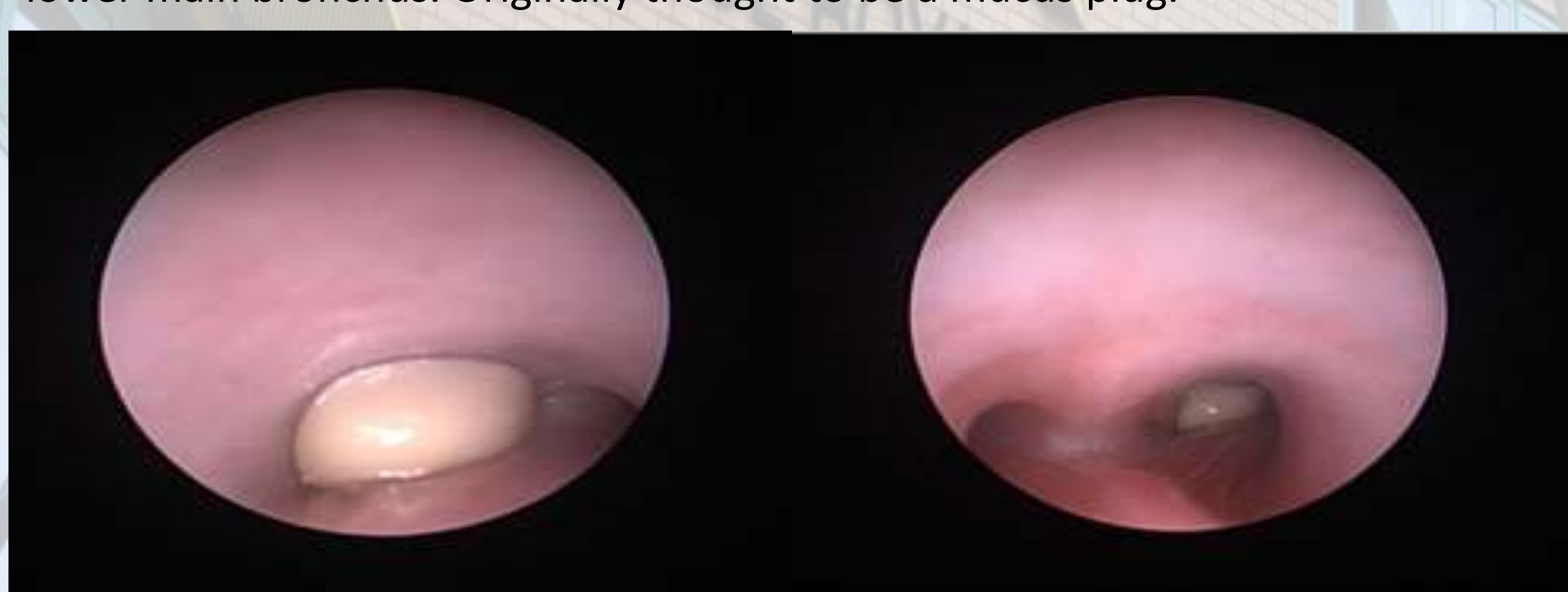

Image 3: Endoscopic images from the rigid bronchoscopy demonstrating a pedunculated mass arising from the wall of the right main bronchus

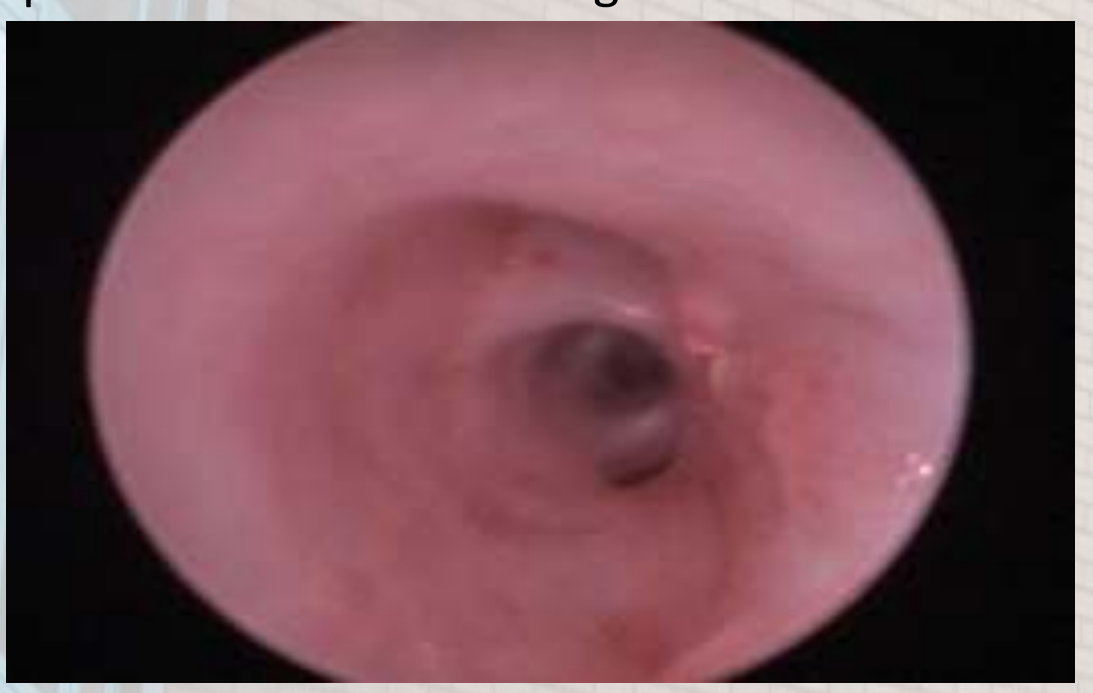

Image 4: Appearance of he right main bronchus after removal of lesion

\section{Summary}

Trauma from suctioning can cause significant morbidity in both intubated patients and tracheostomised patients. In this case the resulting granulation led to unilateral hypoventilation and collapse. It is important that intensive care staff are aware of the importance of suctioning to the correct depth.

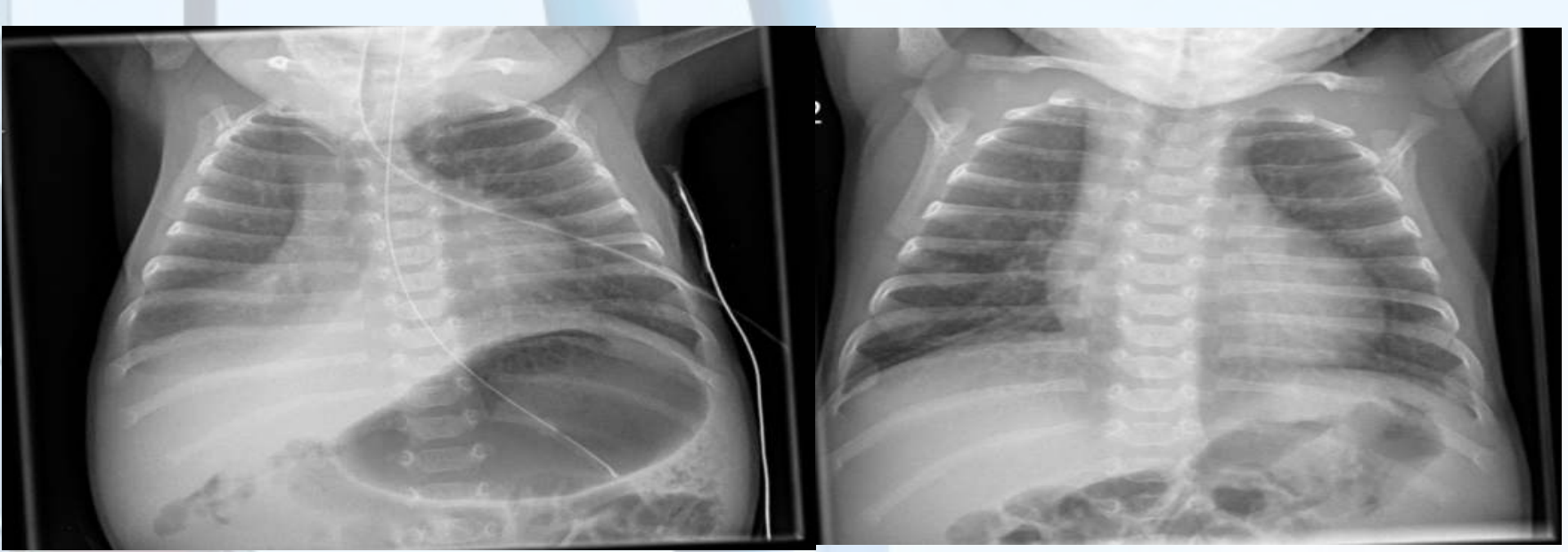

Image 1: Chest x-ray's showing pre and post excision of lesion. The left image shows mid and lower lobe collapse. This is resolved in the post excision $x$-ray on the right

A 28 week year old premature male twin was transferred intubated (orotracheal intubation) to Bristol Royal Children's hospital from an outlying unit. Prior to transfer he had been intubated at birth and noted to have a large right pneumothorax. This had been managed with a chest drain and had resolved. He had been jaundiced and had suffered a small intraventricular haemorrhage (grade 2). Both of these had resolved.

He was extubated at day 3 to high flow oxygen then at day 11 to low flow oxygen. At day 12 he suffered frequent desaturations and bradycardia and then required CPAP. He moved between CPAP and high flow for a further 21 days. At day 33 of life the baby's work of breathing increased significantly. Serial chest $x$-rays demonstrated right upper lobe hyperinflation, along with right middle and lower lobe collapse (image 1). This was though to be a right congenital pneumonia.

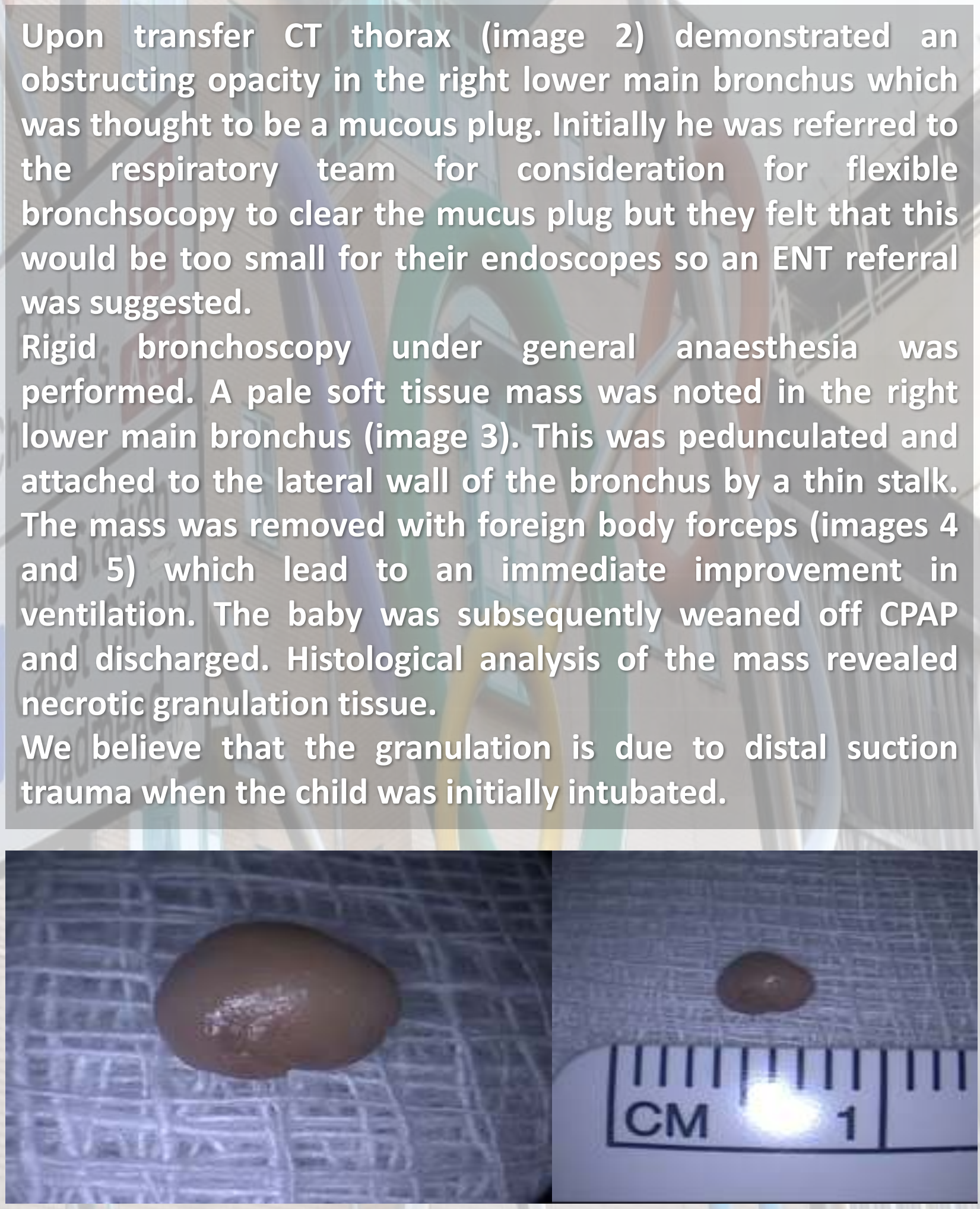

Image 5: The excised granuloma measuring $3.5 \mathrm{~mm}$ 